\begin{tabular}{|c|l|}
\hline Title & Specific antibody responses in dogs experimentally infected with Echinococcus multil ocularis \\
\hline Author(s) & TANA KA, Rika \\
\hline Citation & Japanese Journal of Veterinary Research, 46(2-3), 138-139 \\
\hline Issue Date & 1998-11-30 \\
\hline Doc URL & http://hdl.handle.net/2115/2688 \\
\hline Type & bulletin (article) \\
\hline File Information & KJo0003408021.pdf \\
\hline
\end{tabular}

Instructions for use 
specifically and strongly reacted with sera from $B$. caballi-, but not $B$. equi-infected and uninfected horses. Therefore, B.cA2 product is thought to be suitable for serodiagnosis of equine babesiosis caused by $B$. caballi.

Antigenic diversities among strains cause problems in specificity and sensitivity of serodiagnosis of many protozoan diseases. However,
RAP-1 molecules of other Babesia species have been reported to be species-specific antigens. Therefore, B.cA2 is thought to contain speciesspecific epitopes among strains. ELISA based on B.cA2 recombinant product will be developed after fine analysis of antigenic homogeneity of this molecule among $B$. caballi stocks.

\title{
Specific antibody responses in dogs experimentally infected with Echinococcus multilocularis.
}

\author{
Rika Tanaka \\ Laboratory of Parasitology, \\ Department of Disease Control, \\ School of Veterinary Medicine, \\ Hokkaido University, Sapporo 060-0818, Japan
}

Specific serum IgG, IgA and IgM, and copro IgA against somatic and excretory/secretory antigens of adult tapeworms and protoscolices of Echinococcus multilocularis were analyzed in dogs experimentally infected with $E$. multilocularis by enzyme-linked immunosorbent assay (ELISA).

First, two mongrel dogs were infected with E. multilocularis and their sera and fecal samples were collected until 33 days post infection (DPI). IgG and IgA responses against adult antigens showed a gradual increase from 12 DPI whereas IgM against adult antigens showed a transitory rise in one dog. Similar time course of responses against protoscolex antigens were observed, but the responses were weak. IgG against EmA9 (designated by Khono et al., 1995)-antigen which specifically reacts with monoclonal antibody, EmA9, used in coproantigen detection assay also showed a gradual increase from $12 \mathrm{DPI}$, indicates host immune system recognized EmA9-antigen excreted in the host feces. Western blot analysis of adult and protoscolex antigens with sera of $E$. multilocular- is infected dogs showed distinct band patterns in IgG and IgA reactions.

Secondly, four beagle dogs were divided into two groups, two dogs into reinfecion group which were infected with $E$. multilocularis and purged at $21 \mathrm{DPI}$, reinfected at $35 \mathrm{DPI}$, and the other two into control group which were infected with E. multilocularis at $35 \mathrm{DPI}$, the same time as reinfection in reinfection group. Sera and fecal samples were collected until the autopsy ( 80 days after reinfection). IgG and IgA against adult antigens and IgG against EmA9 antigen showed an evident increase from 14 or 15 days post primary infection in both groups. Higher titer was correlated with long-standing infections. Rapid and strong IgG responses after reinfection were not observed. Time course of coproantigen excretion was also examined by sandwich ELISA using monoclonal antibody EmA9. The peak of the OD value in reinfecion group was less than that in control group and the decrease of the $O D$ value after the peak was more rapid in reinfection group. The number of worms recovered in reinfection group at the autopsy were 
220 and 0 whereas 12,310 and 2,804 in control group.

Finally, two beagle dogs were infected with E. multilocularis, and their sera and fecal samples were collected until 21 DPI for the detection of copro IgA and serum IgA against adult and protoscolex antigens. Serum IgA against adult antigens were detected at $21 \mathrm{DPI}$, but copro IgA against adult or protoscolex antigens were not detected. Immune complex consisted with both copro IgA and antigen were not detected.

Those results showed that the definitive hosts recognized worm excretory/secretory product or worm somatic material and produced specific IgG and IgA. The rapid and strong antibody response was not clearly observed at reinfection, but worm expulsion was promoted in reinfection group.

Improvement of the alternative definitive host model for Echinococcus multilocularis in adrenocorticosteroid treatment for Mongolian gerbil, Meriones unguiculatus

\author{
Eiichi Miwa \\ Laboratory of Parasitology, \\ Department of Disease Control, \\ School of Veterinary Medicine, \\ Hokkaido University, Sapporo 060-0818, Japan
}

The effect of the different treatment of adrenocorticosteroids on the alternative definitive host model for Echinococcus multilocularis was evaluated using Mongolian gerbil (Meriones unguiculatus).

First, the effect of injection route of prednisolone tertiary-butylacetate (PTBA), one is subcutaneous (s.c.) and the other intraperitoneal (i.p.), was compared. More parasites were recovered from s.c. group than i.p. group.

Secondly, the effect of oral administration of prednisolone and betamethasone was compared to s.c. of PTBA. Most parasites were recovered from PTBA s.c. group. Scarce effects of oral administration of prednisolone and betamethasone were observed not only in the number of parasite recovered but also in the plasma biochemical parameters and the number of peripheral WBC and lymphocytes.

Thirdly, the effect of intervals of PTBA s.c. injection was evaluated by comparing once per 1 day, once per 2 days and once per 3 days. More parasites were recovered from groups of once per 1 day and once per 2 days than that of once per 3 days.

Lastly, the effect of period of PTBA s.c. injection schedule was evaluated by comparing the injection period from 6 days pre-inoculation to the day of inoculation $(-6 \sim 0)$, from 6 days pre-inoculation to 6 days post-inoculation ( $-6 \sim$ 6 ), from 6 days pre-inoculation to 12 days post-inoculation $(-6 \sim 12)$ and from 6 days preinoculation to 18 days post-inoculation $(-6 \sim 18)$. Less parasites were recovered from $-6 \sim 0$ group than other 3 groups ( $-6 \sim 6$ group, $-6 \sim$ 12 group and $-6 \sim 18$ group), indicating that PTBA administration until 6 days post-inoculation was important for the parasite initial establishment. Analysis of change in the coproantigen excretion during the experimental infection and analysis of the parasite size recovered at necropsy indicated that $-6 \sim 18$ administration was most efficient for the parasite growth.

To evaluate the specific effect of PTBA on 